\title{
Detection of Vibration-Mode Scattering in Electronic Shot Noise
}

\author{
Manohar Kumar, ${ }^{1}$ Rémi Avriller, ${ }^{2,3,4}$ Alfredo Levy Yeyati, ${ }^{2}$ and Jan M. van Ruitenbeek ${ }^{1}$ \\ ${ }^{1}$ Kamerlingh Onnes Laboratorium, Universiteit Leiden, PO Box 9504, 2300 RA Leiden, The Netherlands \\ ${ }^{2}$ Departamento de Física Teórica de la Materia Condensada C-V, Universidad Autónoma de Madrid, E-28049 Madrid, Spain \\ ${ }^{3}$ Donostia International Physics Center (DIPC), Paseo Manuel de Lardizabal 4, E-20018 San Sebastian, Spain \\ ${ }^{4}$ Univ. Bordeaux, LOMA, UMR 5798, F-33400 Talence, France and CNRS, LOMA, UMR 5798, F-33400 Talence, France
}

(Received 14 September 2011; published 5 April 2012)

\begin{abstract}
We present shot noise measurements on $\mathrm{Au}$ nanowires showing very pronounced vibration-mode features. In accordance to recent theoretical predictions the sign of the inelastic signal, i.e., the signal due to vibration excitations, depends on the transmission probability becoming negative below a certain transmission value. We argue that the negative contribution to noise arises from coherent two-electron processes mediated by electron-phonon scattering and the Pauli exclusion principle. These signals can provide unique information on the local phonon population and lattice temperature of the nanoscale system.
\end{abstract}

DOI: 10.1103/PhysRevLett.108.146602

Many properties of electronic conductors become fundamentally modified at the nanoscale. For example, the effects of lattice vibrations on electron transport in bulk metals is only visible as a smooth temperature dependence of the resistivity. At the nanoscale, on the other hand, individual atomic and molecular vibration modes are revealed in the differential conductance [1-3]. Here, we address another phenomenon that becomes uncovered at the nanoscale: the excitation of discrete atomic vibrations appearing in electronic shot noise. Shot noise is white noise that arises from fluctuations in the occupation numbers of quasiparticle states in a conductor. For a tunnel junction the noise power is given by $S_{I}=2 e\langle I\rangle$, where $e$ is the electron charge and $\langle I\rangle$ is the time-averaged current. We will be considering nanoscale systems for which electron-electron interaction effects are negligible. In such systems shot noise is reduced compared to the tunnel limit due to the coherence between the electron states of the two leads [4-8]. For a system that has $N$ conductance channels, with each channel $n$ characterized by a transmission probability $\tau_{n}$, the conductance and the shot noise power can be expressed as $G=G_{0} \sum_{n=1}^{N} \tau_{n}$ and [8]

$$
\begin{aligned}
S_{I}= & 2 e V G_{0} \operatorname{coth}\left(\frac{\mathrm{eV}}{2 k_{B} T}\right) \sum_{n=1}^{N} \tau_{n}\left(1-\tau_{n}\right) \\
& +4 k_{B} T G_{0} \sum_{n=1}^{N} \tau_{n}^{2}
\end{aligned}
$$

respectively, where $G_{0}=2 e^{2} / h$ is the quantum of conductance, $V$ is the applied bias potential, and $k_{B} T$ is the thermal energy. Equation (1) combines the JohnsonNyquist thermal noise with the noise due to the nonequilibrium electron distribution at finite bias. For $k_{B} T \ll \mathrm{eV}$ it reduces to $S_{I}=2 e\langle I\rangle F$ where the Fano factor $F$ is given by
PACS numbers: 72.70.+m, 73.63.Rt, 72.10.Di, 85.65.+h

$$
F=\frac{\sum_{n=1}^{N} \tau_{n}\left(1-\tau_{n}\right)}{\sum_{n=1}^{N} \tau_{n}} .
$$

Recently, several groups have predicted that nontrivial deviations should appear in this linear dependence of shot noise on the average current, at bias voltages exceeding the threshold for excitation of vibration modes [9-11]. As a first test system for the investigation of inelastic contributions to shot noise we have selected $\mathrm{Au}$ atomic chains: a nanoscale system that is easily reproduced, has just a single conductance channel, and low-lying vibration modes $[2,12-14]$. The atomic chains are formed using a mechanically controllable break junction technique [13], by gently breaking an atomic-scale contact between gold wires.

The electronic circuit for the measurement is shown in Fig. 1(a). The Au atomic contact was first characterized by recording conductance and length histograms $[12,13,15]$. For details of the experimental procedure we refer to the Supplemental Material [16]. After this initial characterization atomic chains of 3 to 5 atoms in length were formed for the analysis of shot noise. Figure 1(b) shows the results for the Fano factor for many of such atomic chains as measured for bias voltages below $10 \mathrm{mV}$, and plotted against the conductance as done for instance in Ref. [4]. Most atomic chain junctions have a conductance slightly below $1 G_{0}$, but somewhat smaller values frequently occur because of scattering on defects in the leads [17,18]. All data in Fig. 1(b) fall close to the solid line, which is the expected dependence for a single conductance channel, as given by Eqs. (1) and (2).

The phonon signals in the conductance were measured for each junction by recording the differential conductance, $d I / d V$, using a lock-in technique, an example of which is shown in Fig. 1(c). Although the Au atomic chain permits many vibration modes, only the longitudinal mode with largest momentum has a significant cross section for 

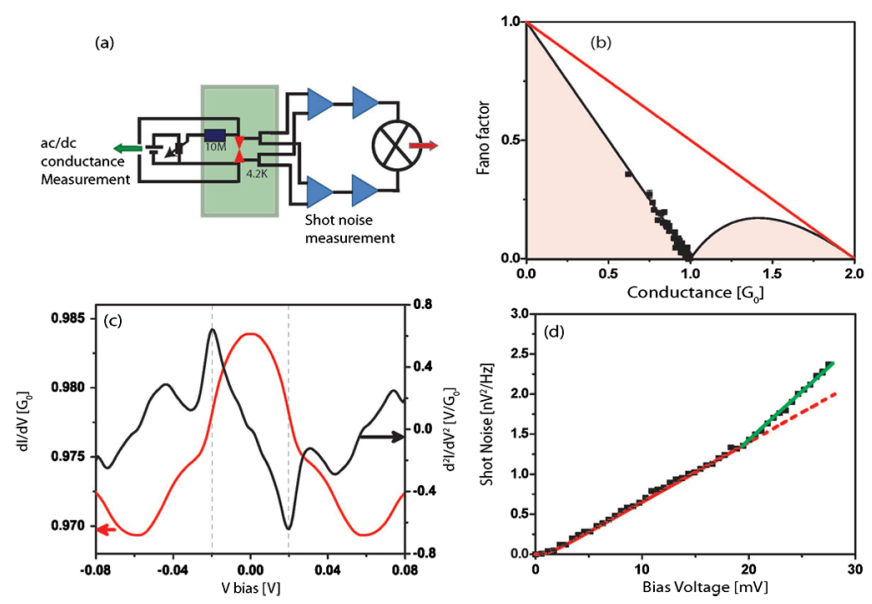

FIG. 1 (color online). Measurement of inelastic signals in shot noise for Au atomic chains. (a) Circuit diagram for the measurement of conductance and noise on atomic junctions formed by the mechanically controllable break junction technique. Noise is measured by employing two sets of low-noise amplifiers in parallel, each having an amplification factor of $10^{5}$. A spectrum analyzer is used for taking the cross spectrum of the two channels, in a frequency range of $250 \mathrm{~Hz}$ to $100 \mathrm{kHz}$. By averaging over $10^{4}$ spectra the uncorrelated noise of the preamplifiers is suppressed. (b) Fano factor plot for many different realizations of atomic chains. The data are consistent with assuming a single conductance channel only. (c) Symmetric part of the differential conductance (left axis) and its derivative (right axis) for a Au atomic chain. The signal was measured with $2 \mathrm{mV}$ modulation amplitude at $3.33 \mathrm{kHz}$; the second derivative was computed numerically. The peak at $20.0 \pm 0.4 \mathrm{mV}$ signals the onset of scattering by a vibration-mode. (d) Shot noise as a function of bias for the same atomic contact as in (c). The red (medium gray) curve is a fit to Eq. (1) up to $20 \mathrm{mV}$. The Fano factor $F_{1}=0.020 \pm 0.002$ agrees with the conductance of $G=0.98 G_{0}$ in (c). The broken red curve is an extrapolation of the fit. The green (light gray) line is a linear fit to the data above $20 \mathrm{mV}$.

inelastic scattering [2]. The scattering on the vibration mode is seen as a drop of about $1 \%$ in the conductance at about $20 \mathrm{mV}$, which agrees well with the expected vibration-mode energy. The vibration mode can attain values between about $10 \mathrm{mV}$ and $20 \mathrm{mV}$, which varies due to softening of the bonds with the strain in the atomic wire [2]. In addition to the vibration-mode signal one observes a background of irregular conductance fluctuations, which is attributed to interference of electron trajectories as a result of scattering on defects in the leads $[17,18]$. By taking the symmetrized spectrum, $[G(V)+G(-V)] / 2$, this background can be partly removed.

Figure 1(d) shows a measurement of the noise power for the same contact as in Fig. 1(c), measured up to $28 \mathrm{mV}$. The small curvature at low bias is due to the crossover from thermal to nonequilibrium noise. Above $3 \mathrm{mV}$ the noise power closely follows the expected linear dependence. The red (medium gray) curve is a fit to Eq. (1) and gives a Fano factor $F_{1}=0.020 \pm 0.002$, which agrees with the zero-bias conductance of $G=0.98 G_{0}$ for a single channel, within the accuracy of $1 \%$ in $G$.

Above $20 \mathrm{mV}$ we observe a sudden kink in the noise signal, which matches the energy of the vibration mode seen in Fig. 1(c). If we take the slope above the kink to define a modified Fano factor $F_{2}$ the relative change in Fano factor is given as $\delta F / F=\left(F_{2}-F_{1}\right) / F_{1}=+0.90$. We interpret this kink as evidence for an inelastic scattering contribution to shot noise. This interpretation was further tested, and the results are discussed below.

Before presenting the full data set we note that the analysis is simplified by introducing the reduced noise, given as

$$
Y(V)=\frac{S_{I}(V)-S_{I}(0)}{S_{I}(0)} .
$$

Further, we introduce the parameter

$$
X(V)=\frac{\mathrm{eV}}{2 k_{B} T} \operatorname{coth}\left(\frac{\mathrm{eV}}{2 k_{B} T}\right),
$$

which reduces the expression for the noise in (1) to a simple linear relationship $Y(V)=[X(V)-1] F$. All dependence on the transmission probabilities is thus lumped into the Fano factor $F$ of Eq. (2).

Figure 2(a) shows data for seven different chain configurations, plotted as $Y(V)$ vs $X(V)$. For $G$ close to $1 G_{0}$ (lowest curves) we find a positive correction above the kink. Note the curve with $G=1.00 G_{0}$, which shows zero noise $(F=0)$ until a kink appears when reaching the vibration-mode energy. Remarkably, for $G<0.95 G_{0}$ the correction above the kink has the opposite sign. The observed dependence is linear in $X(V)$, above and below the kinks, within the experimental accuracy. In some cases we observe a second kink at still higher bias voltages (not shown here), but for the purpose of this Letter we limit the analysis to the first kink only.

Figure 2(b) shows the position of the steps in the differential conductance against the observed position of the kink in the reduced-axis plot. The uncertainty and the scatter in the mode energy are due to the difficulty of removing the contributions by conductance fluctuations from the differential conductance. Despite the large uncertainties, a clear trend is observed and the data lie close to the line 1:1. This observation provides strong support for the interpretation of the kink in the noise data as being due to inelastic scattering on vibration modes of the system.

The relative change in the Fano factor for a set of about 120 measurements is shown in Fig. 3, plotted as a function of the transmission probability $\tau$. For $\tau$ close to 1 exclusively positive values for $\delta F / F$ are found, while for $\tau<$ 0.95 only negative values occur, confirming the trend observed in Fig. 2(a).

While the effect of inelastic scattering on vibration modes in atomic-size systems has been extensively analyzed theoretically [19-22] the generalization of these studies to noise properties has only recently started 

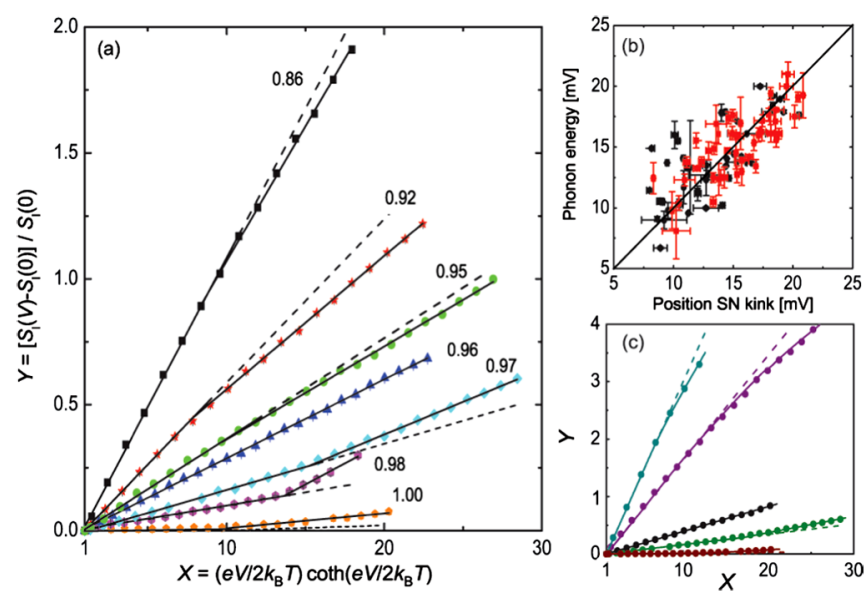

FIG. 2 (color online). The kink in shot noise and its sign and position for a range of values of the transmission of the contact. (a) Reduced noise $Y$, Eq. (3), plotted as a function of the variable $X$, defined in Eq. (4). The data below the phonon energy are described by a linear dependence (fitted with a solid line up to the kink, and extrapolated by a dashed line). The slope of this line gives the Fano factor. The transmission probability obtained from the conductance is shown as a label to each curve. Above the phonon energy a new linear dependence is observed. The kink is towards higher slope when the transmission probability $\tau$ is close to 1 . For $\tau$ below 0.94 we observe a reduced slope. (b) The phonon energy as observed in the differential conductance plotted as a function of the position of kink in the shot noise. The line shows the expected 1:1 relation. Red and black points are for positive and negative kinks, respectively. (c) Fits of the theory, including the energy dependence of the transmission as estimated from the $d I / d V$ signals (full curves). The broken curves show the linear extrapolation of the zero-bias curve. The values for the zero-bias transmission $\tau$ and the inelastic scattering strength are (from top to bottom): $\tau=0.669, \lambda=0.35 \Gamma$; $\tau=0.818, \lambda=0.20 \Gamma ; \tau=0.956, \lambda$ undetermined; $\tau=0.981$, $\lambda=0.11 \Gamma ; \tau=0.998, \lambda=0.08 \Gamma$.

[9-11]. Most of these studies are based on a minimal model corresponding to a single resonant molecular level $E_{d}$ coupled to the left and right leads by tunneling rates, $\Gamma_{L}$ and $\Gamma_{R}$, interacting with a localized vibration mode with frequency $\omega_{0}$ and a coupling parameter $\lambda$. This model, schematically depicted in the inset of Fig. 3 , is adequate for representing a nanosize junction in the regime $\Gamma_{L, R} \gg \omega_{0}$.

The effect of the phonon mode in the electronic noise properties has been analyzed up to second order in $\lambda$, which is reasonable when the step in the conductance due to inelastic scattering is at most a few percent, see Fig. 1(b). When the energy dependence of the transmission can be neglected, $\tau(E) \simeq \tau(0)$, the analysis simplifies, and for the zero temperature and positive voltages, the inelastic correction to the noise becomes [9]

$$
\begin{aligned}
\delta S_{I} \simeq & \frac{e^{2}}{h}\left(\frac{\lambda}{\Gamma}\right)^{2} \tau^{2}\{2(1-\tau)(1-2 \tau) \mathrm{eV} \\
& \left.+\left(8 \tau^{2}-8 \tau+1\right)\left(\mathrm{eV}-\hbar \omega_{0}\right) \theta\left(\mathrm{eV}-\hbar \omega_{0}\right)\right\}
\end{aligned}
$$

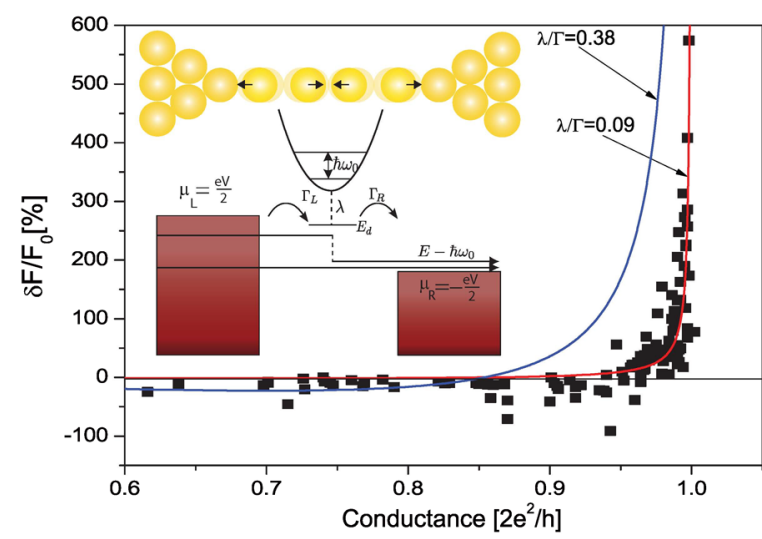

FIG. 3 (color online). Distribution of the observed change in the Fano factor at the kink, plotted as a function of the conductance. The points are obtained for different realizations of $\mathrm{Au}$ atomic chains. For conductance (i.e., transmission) close to 1 we find exclusively positive values for $\delta F / F$. Below a cross-over regime near $G=0.95 G_{0}$ only negative values are observed. The curves are obtained from the theory of Ref. [9], for fixed values of the inelastic scattering strength $\lambda$. Inset: The top part illustrates the atomic chain configuration and the vibration mode involved in the scattering. The lower part sketches an example of a two-electron process giving rise to reduction of the Fano factor: two electrons injected from the left lead at different energies tend to compete for the same outgoing state after the emission of a phonon (see text).

where the transmission probability at the Fermi energy is given by $\tau=4 \Gamma_{L} \Gamma_{R} /\left(E_{d}^{2}+\Gamma^{2}\right)$ with $\Gamma=\Gamma_{L}+\Gamma_{R}$. This expression clearly predicts a crossover from a positive to a negative correction in the noise as the transmission is reduced from the unitary limit.

A positive correction due to inelastic scattering giving additional shot noise, such as observed for $\tau$ close to 1 , is quite intuitive. However, a negative correction such as observed for lower transmission values cannot be understood in simple terms. Equation (5) indicates that the crossover from positive to negative correction is predicted at $\tau_{ \pm}=1 / 2 \pm 1 / 2 \sqrt{2}$. The higher crossover point, relevant for the present data set, is $\tau_{+} \simeq 0.854$. The microscopic processes contributing to inelastic noise in Eq. (5) can be classified into one-electron and two-electron processes, i.e., $\delta S^{(i n)}=\delta S_{1 e}^{(i n)}+\delta S_{2 e}^{(i n)}$ (see the Supplemental Material [16]). While $\delta S_{1 e}^{(i n)}$ scales as $\tau^{2}$ and is thus always positive, the two-electron contribution $\delta S_{2 e}^{(i n)}$ scales as $-8 \tau^{3}(1-\tau)$ and thus it is always negative. This behavior can be qualitatively understood as arising from the Pauli principle as illustrated by the diagram in the inset of Fig. 3. In this diagram two electrons are injected from the left lead with energies $E$ and $E-\hbar \omega_{0}$ within the interval $(-V / 2,+V / 2)$. If the higher energy electron emits a phonon it would tend to occupy the same outgoing state as the other electron. This process would thus be blocked at perfect transmission. Additionally, this process tends to 
reduce the noise by narrowing the energy distribution of the outgoing electrons.

The solid curves in Fig. 3 show the calculated dependence of $\delta F / F$ as a function of $\tau$ for two fixed choices of electron-vibron scattering strength $\lambda$. The variation of the data can be largely attributed to variations in $\lambda$ for different chain configurations. There appears to be a trend of $\lambda$ growing when $\tau$ decreases below 1 . The crossover in the data to negative values for $\delta F$ appears at higher $\tau$ than expected. The fluctuations in the background of the differential conductance indicate that our assumption of an energy independent transmission may need to be relaxed. Such energy dependence modifies the theory for inelastic scattering but may also affect the linearity of the Fano plots directly, i.e., the usual elastic component of the noise. We have used the measured differential conductance curves to estimate the size of this energy dependence, and have calculated the bias dependence of the noise numerically. Figure 2(c) shows fits to representative data sets (see also the Supplemental Material [16]). It demonstrates that the curves can be correctly described with reasonable values for the parameters, and that the corrections due to the energy dependence of $\tau$ to the elastic noise are modest in these cases (see Fig. 5 in the Supplemental Material [16]).

This experiment is a first step towards a more quantitative study of phonon statistics in molecular electronics $[7,23]$. The coupling of atomic vibrations and electronic noise can be further exploited for the determination of the lattice temperature in molecular junctions under an applied bias, an idea which is the subject of recent theoretical speculation [24-26].

We thank Roel Smit, Kiran Kumar, and Zhengpen Baardman for their assistance in the experiments, and Bert Crama and Ruud van Egmond for expert technical support. Fruitful discussions with Thomas Frederiksen, Luis Foa Torres, F. Haupt, and D. Urban are acknowledged. This work is part of the research program of the Foundation for Fundamental Research on Matter (FOM), which is financially supported by the Netherlands Organization for Scientific Research (NWO). It was further supported by the Spanish MICINN under Contract No. NAN2007-29366-E (Era-Net project CHENANOM).

[1] B. C. Stipe, M. A. Rezaei, and W. Ho, Science 280, 1732 (1998).

[2] N. Agraït, C. Untiedt, G. Rubio-Bollinger, and S. Vieira, Phys. Rev. Lett. 88, 216803 (2002).
[3] R. H. M. Smit, Y. Noat, C. Untiedt, N. D. Lang, M. C. van Hemert and J. M. van Ruitenbeek, Nature (London) 419, 906 (2002).

[4] A. Kumar, L. Saminadayar, D. C. Glattli, Y. Jin, and B. Etienne, Phys. Rev. Lett. 76, 2778 (1996).

[5] M. Reznikov, M. Heiblum, H. Shtrikman, and D. Mahalu, Phys. Rev. Lett. 75, 3340 (1995).

[6] H. E. van den Brom and J. M. van Ruitenbeek, Phys. Rev. Lett. 82, 1526 (1999).

[7] D. Djukic and J. M. van Ruitenbeek, Nano Lett. 6, 789 (2006).

[8] Ya. M. Blanter and M. Büttiker, Phys. Rep. 336, 1 (2000).

[9] R. Avriller and A. Levy Yeyati, Phys. Rev. B 80, 041309 (R) (2009).

[10] F. Haupt, T. Novotný and W. Belzig, Phys. Rev. Lett. 103, 136601 (2009).

[11] T. L. Schmidt and A. Komnik, Phys. Rev. B 80, 041307(R) (2009).

[12] A. I. Yanson, G. Rubio Bollinger, H. E. van den Brom, N. Agraït, and J. M. van Ruitenbeek, Nature (London) 395, 783 (1998).

[13] N. Agraït, A. Levy Yeyati and J. M. van Ruitenbeek, Phys. Rep. 377, 81 (2003).

[14] H. Ohnishi, Y. Kondo, and K. Takayanagi, Nature (London) 395, 780 (1998).

[15] R.H.M. Smit, C. Untiedt, A.I. Yanson, and J.M. van Ruitenbeek, Phys. Rev. Lett. 87, 266102 (2001).

[16] See Supplemental Material at http://link.aps.org/ supplemental/10.1103/PhysRevLett.108.146602 for details on the experimental procedure and for the theoretical analysis of one-electron and two-electron processes.

[17] B. Ludoph, M. H. Devoret, D. Esteve, C. Urbina, and J. M. van Ruitenbeek, Phys. Rev. Lett. 82, 1530 (1999).

[18] C. Untiedt, G. R. Bollinger, S. Vieira, and N. Agrait, Phys. Rev. B 62, 9962 (2000).

[19] L. de la Vega, A. Martin-Rodero, N. Agrait, and A. LevyYeyati, Phys. Rev. B 73, 075428 (2006).

[20] T. Frederiksen, M. Brandbyge, N. Lorente, and A.P. Jauho, Phys. Rev. Lett. 93, 256601 (2004).

[21] M. Paulsson, T. Frederiksen, H. Ueba, N. Lorente, and M. Brandbyge, Phys. Rev. Lett. 100, 226604 (2008).

[22] R. Egger and A. O. Gogolin, Phys. Rev. B 77, 113405 (2008).

[23] M. Kiguchi, O. Tal, S. Wohlthat, F. Pauly, M. Krieger, D. Djukic, J. C. Cuevas, and J. M. van Ruitenbeek, Phys. Rev. Lett. 101, 046801 (2008).

[24] Y.-C. Chen and M. Di Ventra, Phys. Rev. Lett. 95, 166802 (2005).

[25] D. F. Urban, R. Avriller, and A. Levy Yeyati, Phys. Rev. B 82, 121414(R) (2010).

[26] T. Novotný, F. Haupt, and W. Belzig, Phys. Rev. B 84, 113107 (2011). 